$01,10,12$

\title{
Изучение свойств сплава золото-железо в макро- и нанокристаллических состояниях в различных $P-T$-условиях
}

\author{
(C) М.Н. Магомедов
}

Институт проблем геотермии и возобновляемой энергетики филиал Объединенного института высоких температур РАН, Махачкала, Россия

E-mail: mahmag4@mail.ru

Поступила в Редакцию 21 августа 2020 г.

В окончательной редакции 21 августа 2020 г.

Принята к публикации 27 августа 2020 г.

Для неупорядоченного сплава замещения ГЦК-Au-Fe определены параметры парного межатомного потенциала Ми-Леннард-Джонса. На основе данных параметров рассчитаны концентрационные зависимости решеточных свойств для макрокристалла данного сплава. Расчеты 20 свойств макрокристаллов ГЦК-Аи, ГЦК-Fе и ГЦК- $\mathrm{Au}_{0.5} \mathrm{Fe}_{0.5}$ показали хорошее согласие с экспериментальными данными. Используя RР-модель нанокристалла, рассчитано уравнение состояния $P(v, T ; N)$ и барические зависимости как решеточных, так и поверхностных свойств сплава ГЦК- $\mathrm{Au}_{0.5} \mathrm{Fe}_{0.5}$. Расчеты были проведены при температурах $T=100$, 300 и $500 \mathrm{~K}$ как для макрокристалла $(N=\infty)$, так и для нанокристалла кубической формы из $N=306$ атомов. Показано, что при изотермо-изобарическом $(P=0)$ уменьшении размера нанокристалла его температура Дебая, модуль упругости и удельная поверхностная энергия уменьшаются, а его удельный объем, коэффициент теплового расширения, удельная теплоемкость и коэффициент Пуассона увеличиваются. При низких температурах в определенной области давлений удельная поверхностная энергия возрастает при изотермо-изобарическом уменьшении числа атомов в нанокристалле. С ростом температуры эта область давлений исчезает.

Ключевые слова: золото, железо, сплав замещения, нанокристалл, уравнение состояния, модуль упругости, тепловое расширение, поверхностная энергия.

DOI: 10.21883/FTT.2020.12.50206.172

\section{1. Введение}

Твердый раствор замещения $\mathrm{Au}-\mathrm{Fe}$ имеет уникальные физические свойства, которые востребованы как в науке, так и в технике [1-3]. Благодаря исключительной биосовместимости и благоприятным физическим свойствам наночастицы $\mathrm{Au}-\mathrm{Fe}$ находят различные применения и в медицинских науках [4]. Именно поэтому этот сплав активно изучается как экспериментально $[1-3,5,6]$, так и теоретически [7-10].

Смеси атомов $\mathrm{Au}$ и Fе встречаются редко потому, что эти элементы не смешиваются при низких температурах. Но при высоких температурах, как экспериментально установлено, богатые золотом сплавы Au-Fe образуют твердый раствор замещения с гранецентрированной кубической (ГЦК) структурой $[5,6]$. И хотя ГЦК-структура является высокотемпературной фазой $\mathrm{Fe}$, было показано, что сплавы $\mathrm{Au}_{1-C} \mathrm{Fe}_{C}$ с содержанием железа до $C<53 \%$ легко стабилизируются вплоть до комнатной температуры $[6,7]$. Несмотря на то, что сплавы $\mathrm{Au}-\mathrm{Fe}$ изучаются давно, но термодинамические свойства данного сплава изучены мало. И в литературе нет данных ни для уравнения состояния, ни для поверхностной энергии даже для макрокристаллов $\mathrm{Au}_{1-C} \mathrm{Fe}_{C}[7,8]$.

Что касается свойств нанокристаллов $\mathrm{Au}_{1-C} \mathrm{Fe}_{C}$, то основная часть работ посвящена изучению структурных и магнитных свойств данного сплава $[1,2,6,8-10]$, и очень мало работ посвящено изучению как уравнения состояния, так и термодинамических свойств сплава $\mathrm{Au}_{1-C} \mathrm{Fe}_{C}$ в нанокристаллическом состоянии.

Это обусловлено тем, что экспериментальное изучение размерных зависимостей свойств нанокристалла (как однокомпонентного, так и многокомпонентного вещества) проводят, как правило, в изобарических условиях, т.е. когда давление в системе постоянно: $P=$ const [1-3]. Вместе с тем теоретические расчеты проводят, в большинстве работ, для изохорического процесса, т. е. при постоянной плотности нанокристалла: $R=$ const [8-10]. Это связано с тем, что в эксперименте трудно сохранить постоянной плотность нанокристалла, а в теоретических моделях трудно было определить поверхностное давление, а потому и уравнение состояния нанокристалла.

Разработанная нами в $[11,12]$ методика расчета позволяет рассчитать, как уравнение состояния нанокристалла, так и барическую зависимость его свойств. Поэтому с помощью этой методики нам удалось изучить изменение свойств нанокристалла как при изохорическом $(R=\mathrm{const})$, так и при изобарическом $(P=\mathrm{const})$ изменении размера нанокристалла. Данная методика была первоначально разработана нами для однокомпонентного вещества $[11,12]$, но в дальнейшем она была обобщена в [13] на случай нанокристалла бинарного состава. 
Поэтому в данной работе мы изучим уравнение состояния и барические зависимости различных свойств неупорядоченного твердого раствора замещения ГЦК-Au-Fe на основе методики предложенной в [13]. При этом данные свойства будут изучены как для макрокристалла, так и при изоморфно-изотермическом уменьшении числа атомов в нанокристалле данного сплава.

\section{2. Метод расчета свойств кристалла простого вещества}

Для расчета решеточных свойств нанокристалла однокомпонентного вещества необходимо определить, как потенциал взаимодействия пары его атомов, так и метод расчета, который основан на этом потенциале. Представим парное межатомное взаимодействие в виде потенциала Ми-Леннард-Джонса, который имеет следующий вид:

$$
\varphi(r)=\frac{D}{(b-a)}\left[a\left(\frac{r_{o}}{r}\right)^{b}-b\left(\frac{r_{o}}{r}\right)^{a}\right],
$$

где $D$ и $r_{o}$ - глубина и координата минимума потенциала, $b>a>1$ - численные параметры.

Тогда, используя приближение „взаимодействия только ближайших соседей“ температуру Дебая как функцию первого координационного числа $\left(k_{n}\right)$ и расстояния между центрами ближайших атомов можно определить из выражения [14]:

$$
\Theta\left(k_{n}, c\right)=A_{w}\left(k_{n}, c\right) \xi\left[-1+\left(1+\frac{8 D}{k_{B} A_{w}\left(k_{n}, c\right) \xi^{2}}\right)^{\frac{1}{2}}\right]
$$

Здесь $k_{B}-$ постоянная Больцмана, $k_{n}-$ первое координационное число, которое в нанокристалле зависит как от его размера, так и от формы его поверхности $[11,12], c=\left(6 k_{p} v / \pi\right)^{1 / 3}-$ расстояние между центрами ближайших атомов, $v=V / N-$ удельный объем, $V$ и $N$ - объем и число атомов в нанокристалле, $k_{p}$ - коэффициент упаковки. Функция $A_{w}$ возникает из-за учета энергии „нулевых колебаний“ атомов в кристалле

$$
\begin{gathered}
A_{w}\left(k_{n}, c\right)=K_{R} \frac{5 k_{n} a b(b+1)}{144(b-a)}\left(\frac{r_{o}}{c}\right)^{b+2}, \\
K_{R}=\frac{\hbar^{2}}{k_{B} r_{o}^{2} m}, \quad \xi=\frac{9}{k_{n}(\infty)},
\end{gathered}
$$

где $m$ - масса атома, $\hbar$ - постоянная Планка, $k_{n}(\infty)-$ первое координационное число в объеме нанокристалла.

Если для нанокристалла с геометрической поверхностью Гиббса применимо приближение „взаимодействия только ближайших соседей“, то зависимость энергии нанокристалла от размера и формы будет определяться зависимостью от размера и формы среднего (по нанокристаллу) значения первого координационного числа.
В этом случае, используя для колебательного спектра нанокристалла модель Эйнштейна, для удельной (на атом) свободной энергии Гельмгольца нанокристалла можно принять выражение $[11,12,15]$ :

$$
\begin{aligned}
& f_{H}\left(k_{n}, c, T\right)=\left(\frac{k_{n}}{2}\right) D \cdot U(R)+3 k_{B} \Theta_{E}\left(k_{n}, c\right) \\
& \times\left\{\frac{1}{2}+\left(\frac{T}{\Theta_{E}\left(k_{n}, c\right)}\right) \ln \left[1-\exp \left(-\frac{\Theta_{E}\left(k_{n}, c\right)}{T}\right)\right]\right\},
\end{aligned}
$$

где $T$ - температура, $\Theta_{E}$ - температура Эйнштейна, которая связана с температурой Дебая соотношением [15]: $\Theta=(4 / 3) \Theta_{E}, R=r_{o} / c-$ относительная линейная плотность кристалла, функция потенциальной энергии, в соответствии с (1), равна

$$
U(R)=\frac{a R^{b}-b R^{a}}{b-a}
$$

Положим, что зависимость функции $k_{n}$ от размера и формы нанокристалла с поверхностью Гиббса не зависит от плотности $(R)$ и температуры, а определяется только числом атомов в нанокристалле $(N)$, параметром его формы $(f)$ и постоянными $k_{n}(\infty)$ и $k_{p}$. Тогда из $(4)$ для уравнения состояния $P\left(k_{n}, v, T\right)$ и изотермического модуля упругости $\left(B_{T}\right)$ можно получить $[11,12]$ :

$$
\begin{aligned}
P= & -\left(\frac{\partial f_{H}}{\partial v}\right)_{T} \\
= & {\left[\frac{k_{n}}{6} D \cdot U^{\prime}(R)+3 k_{B} \Theta_{E} \cdot \gamma \cdot E_{w}\left(\frac{\Theta_{E}}{T}\right)\right] \frac{1}{v}, } \\
B_{T}= & -v\left(\frac{\partial P}{\partial v}\right)_{T} \\
= & P\left[\frac{k_{n}}{18} D \cdot U^{\prime \prime}(R)+3 k_{B} \Theta_{E} \cdot \gamma(\gamma-q) \cdot E_{w}\left(\frac{\Theta_{E}}{T}\right)\right. \\
& \left.-3 N k_{B} \cdot \gamma^{2} \cdot T \cdot F_{E}\left(\frac{\Theta_{E}}{T}\right)\right] \frac{1}{v} .
\end{aligned}
$$

Здесь введены следующие функции:

$$
\begin{gathered}
E_{w}(y)=0.5+\frac{1}{[\exp (y)-1]}, \\
F_{E}(y)=\frac{y^{2} \exp (y)}{[\exp (y)-1]^{2}}, \\
U^{\prime}(R)=R\left[\frac{\partial U(R)}{\partial R}\right]=\frac{a b\left(R^{b}-R^{a}\right)}{b-a}, \\
U^{\prime \prime}(R)=R\left[\frac{\partial U^{\prime}(R)}{\partial R}\right]=\frac{a b\left(b R^{b}-a R^{a}\right)}{b-a} .
\end{gathered}
$$


Из формулы (2) легко найти выражения для первого $(\gamma)$, второго $(q)$ и третьего $(z)$ параметров Грюнайзена:

$$
\begin{gathered}
\gamma=-\left(\frac{\ln \Theta}{\partial \ln v}\right)_{T}=\frac{b+2}{6\left(1+X_{w}\right)}, \\
q=\left(\frac{\partial \ln \gamma}{\partial \ln v}\right)_{T}=\gamma \frac{X_{w}\left(1+2 X_{w}\right)}{\left(1+X_{w}\right)}, \\
z=-\left(\frac{\partial \ln q}{\partial \ln v}\right)_{T}=\gamma\left(1+4 X_{w}\right)-2 q \\
=\gamma\left(\frac{1+3 X_{w}}{1+X_{w}}\right)=\frac{(b+2)}{6} \frac{\left(1+3 X_{w}\right)}{\left(1+X_{w}\right)^{2}},
\end{gathered}
$$

где введена функция: $X_{w}=A_{w} \xi / \Theta$, которая определяет роль квантовых эффектов.

Так как температура Дебая (2) не зависит от температуры при изохорическом нагреве, то изохорную и изобарную теплоемкости можно определить в следующем виде $[11,12,15]$ :

$$
C_{v}=3 N \cdot k_{B} \cdot F_{E}\left(\frac{\Theta_{E}}{T}\right), \quad C_{p}=C_{v}\left(1+\gamma \alpha_{p} T\right),
$$

где изобарный коэффициент теплового объемного расширения можно рассчитать по уравнению Грюнайзена [15]:

$$
\alpha_{p}=\frac{\gamma \cdot C_{v}}{V \cdot B_{T}}=\frac{\gamma \cdot C_{v}}{N \cdot B_{T}\left[\pi r_{o}^{3} /\left(6 k_{p}\right)\right]}\left(\frac{v_{o}}{v}\right), \quad v_{o}=\frac{\pi r_{o}^{3}}{6 k_{p}} .
$$

Полученные выражения (2)-(10) позволяют рассчитать зависимость, как уравнения состояния, так и указанных свойств от нормированного объема $v / v_{o}=\left(c / r_{o}\right)^{3}=R^{-3}$ и температуры $T$ для однокомпонентного нанокристалла с заданной структурой (т. е. при данных значениях $k_{n}(\infty)$ и $k_{p}$ ) если известны параметры межатомного потенциала (1) и функциональная зависимость первого координационного числа от размера и формы нанокристалла, т. е. функция $k_{n}(N, f)$.

\section{RР-модель нанокристалла}

Для определения функции $k_{n}(N, f)$ была разработана RP-модель нанокристалла $[11,12]$, суть которой в следующем. Положим, что нанокристалл со свободной поверхностью Гиббса имеет вид прямоугольного параллелепипеда (Rectangular Parallelepiped) с квадратным основанием, ограненного гранями типа (100). Величина $f=N_{p s} / N_{p o}$ - это параметр формы, который определяется отношением числа атомов на боковом ребре $N_{p s}$ к числу атомов на ребре основания $N_{p o}$. Для стержневидной формы $f>1$, для куба $f=1$, для нанокристалла пластинчатой формы $f<1$. Число атомов в нанокристалле, равное: $N=f N_{p o}^{3} / \alpha$, изменяется в пределах: $2^{3} / \alpha \leq N \leq \infty$, где $\alpha=\pi /\left(6 k_{p}\right)$ - параметр структуры.
Ограничение системы поверхностью приводит к обрыву межатомных связей на границе. Поэтому если использовано приближение взаимодействия „только ближайших соседей“, то вместо первого координационного числа $k_{n}(\infty)$ необходимо брать $k_{n}(N, f)$ - среднее (по нанокристаллу) значение первого координационного числа, которое будет зависеть как от $N$, так и от $f$. При этом структуру системы (которая определяется коэффициентом упаковки $k_{p}$ ) полагаем неизменной: $k_{p}=$ const.

B рамках RP-модели зависимость нормированного среднего значения первого координационного числа от размера $(N)$ и формы нанокристалла имеет вид $[11,12]$ :

$$
k_{n}^{*}=\frac{k_{n}(N, f)}{k_{n}(\infty)}=1-Z_{s}(f)\left(\frac{\alpha^{2}}{N}\right)^{1 / 3}
$$

где $k_{n}(\infty)=k_{n}(N=\infty)$ - первое координационное число для макрокристалла,

$$
Z_{s}(f)=\frac{1+2 f}{3 f^{2 / 3}}
$$

Функция формы $Z_{s}(f)$ достигает минимума, равного единице при $f=1$, т. е. для формы куба. Для пластинчатых $(f<1)$ или стержневидных $(f>1)$ форм значение $Z_{s}(f)$ больше единицы. Поэтому функция $k_{n}(f)^{*}$ при любом $N$ имеет максимум при $f=1$, т.е. для наиболее энергетически оптимальной - кубической формы прямоугольного параллелепипеда.

Объем и площадь поверхности для RP-модели равны $[11,12]$ :

$$
V=N_{p o}^{3} f c^{3}=N \alpha c^{3}, \quad \Sigma=6 c^{2} \alpha_{s}(N \alpha)^{2 / 3} Z_{s}(f),
$$

где $\alpha_{s}$ - коэффициент, учитывающий плотность упаковки атомов на грани (т. е. в поверхностном слое) нанокристалла: $\alpha_{s} \simeq \alpha^{2 / 3}$. Легко видеть, что объем нанокристалла $V$ не зависит от формы системы, т. е. от величины $f$. В рамках RР-модели для $\sigma(N, f)$ - удельной (на единицу площади) поверхностной энергии грани (100) нанокристалла, ее изохорной и изобарной производных по температуре и для $P_{s f}-$ поверхностного давления можно получить следующие выражения $[11,12]$ :

$$
\begin{gathered}
\sigma(N, f)=-\frac{k_{n}(\infty) D R^{2}}{12 \alpha^{2 / 3} r_{o}^{2}} L_{E}(N, f) \\
\sigma^{\prime}(T)_{v}=\left(\frac{\partial \sigma}{\partial T}\right)_{c, N, f} \\
=-\frac{3 k_{B} R^{2} \gamma(N, f)}{2 \alpha^{2 / 3}(b+2) r_{o}^{2} k_{n}(N, f)^{*}} F_{E}\left(\frac{\Theta_{E}}{T}\right) \\
\sigma^{\prime}(T)_{P}=\left(\frac{\partial \sigma}{\partial T}\right)_{P, N, f}=\sigma^{\prime}(T)_{v}+v \cdot \alpha_{p}\left(\frac{\partial \sigma}{\partial v}\right)_{T, N, f} \\
=\sigma^{\prime}(T)_{v}-\frac{2}{3} \sigma \cdot \alpha_{p} \cdot \Delta_{p}
\end{gathered}
$$




$$
P_{s f}=\left[\frac{\partial(\sigma \Sigma / N)}{\partial v}\right]_{T, N}=P_{l s}\left(1-\Delta_{p}\right),
$$

где давление Лапласа $P_{l s}$ и введенные функции имеют следующий вид $[11,12]$ :

$$
\begin{gathered}
P_{l s}=\frac{2 \Sigma}{3 V} \sigma=\frac{4 \alpha_{s} Z_{s}(f)}{(\alpha N)^{1 / 3} c} \sigma=4 \alpha_{s} \frac{\left(1-k_{n}^{*}\right)}{\alpha c} \sigma \\
L_{E}(N, f)=U(R)+3 H_{w}(N, T), \\
\Delta_{p}=-\frac{1}{2}\left[\frac{\partial \ln (\sigma)}{\partial \ln (c)}\right]_{T, N, k_{p}, f}=1+\frac{1}{2 L_{E}(N, f)} \\
\times\left\{U^{\prime} R-9\left[q-\gamma \cdot t_{y}\left(\frac{\Theta_{E}}{T}\right)\right] H_{w}(N, T)\right\}, \\
H_{w}(N, T)=\frac{6 \gamma(N, f)}{(b+2)}\left[\frac{k_{B} \Theta_{E}(N, f)}{D k_{n}(N, f)}\right] E_{w}\left(\frac{\Theta_{E}}{T}\right), \\
t_{y}(y)=1-\frac{2 y \exp (y)}{[\exp (2 y)-1]} .
\end{gathered}
$$

Зависимость коэффициента Пуассона $\mu_{p}$ от размера $(N)$ и формы $(f)$ нанокристалла при различных $R-T$ условиях можно рассчитать из соотношения [16]:

$$
\begin{gathered}
\mu_{p}(N, T)=\frac{1}{2}-\frac{1}{48 \cdot X_{s c}(N, T) \cdot[\gamma(N)]^{2}}, \\
X_{s c}(N, T)=\frac{\sigma(N, T)}{c \cdot B_{T}(N, T)} .
\end{gathered}
$$

При положительном значении $P_{s f}$ поверхностное давление сжимает нанокристалл, как это обычно и допускается. Очевидно, что в „термодинамическом пределе“ (т.е. когда $N \rightarrow \infty$ и $V \rightarrow \infty$ при $v=V / N=$ const) функции $P_{l s}$ из (17) и $P_{s f}$ из (16) исчезают, ибо в этом случае: $k_{n}^{*}(N \rightarrow \infty) \rightarrow 1$, а выражения из $(13)-(15)$ преобразуются в формулы для макрокристалла. При $T \rightarrow 0 \mathrm{~K}$ функции из (14) и (15) стремятся к нулю при любом $N$, что согласуется с третьим началом термодинамики в „сильной“ формулировке Планка.

Отметим, что поверхностное давление также можно найти и из выражения (5), как разницу между давлениями, рассчитанными для макро- и нанокристалла:

$$
P_{s f}=P(T, v, N=\infty)-P(T, v, N, f) .
$$

Таким образом, полученная в рамках RP-модели функция $k_{n}(N, f)$ вместе с формализмом из $(2)-(21)$ позволяют рассчитать зависимость всех решеточных и поверхностных свойств от размера и формы нанокристалла при любых (соответствующих твердой фазе) $P-T$-условиях.

\section{4. Метод расчета свойств твердого раствора замещения}

Но для реализации формализма из (2) - (21) необходимо знать параметры потенциала (1). Для однокомпонентных веществ метод самосогласованного определения всех 4-х параметров потенциала (1) был предложен нами в [17,18]. При расчете данных параметров для вещества бинарного состава типа $\mathrm{AB}$, был использован метод „среднего атома“ [13], в котором твердый раствор $\mathrm{A}_{1-C} \mathrm{~B}_{C}$ из $N_{A}$ и $N_{B}$ атомов с разной атомной массой $m_{A}$ и $m_{B}$ моделируется изоструктурным виртуальным кристаллом из $N=N_{A}+N_{B}$ одинаковых „средних“ атомов. Значение $m(C)$ - массы „среднего атома“ такого виртуального вещества будет зависеть от концентрации раствора $\left(C=N_{B} / N\right)$ и рассчитывается, как среднее гармоническое от масс составляющих сплав атомов по формуле

$$
m(C)=\left(\frac{P_{\mathrm{A}}}{m_{\mathrm{A}}}+\frac{P_{B}}{m_{B}}\right)^{-1} .
$$

Входящие в (23) функции $P_{\mathrm{A}}$ и $P_{B}=1-P_{\mathrm{A}}$ это геометрические вероятности обнаружить атом А и В в твердом растворе замещения. Они определяются как вероятность того, что точка, случайно поставленная на линию длинной: $(1-C) r_{o \mathrm{~A}}+C r_{o \mathrm{~B}}$, попадет на отрезки $r_{o \mathrm{~A}}$ или $r_{o \mathrm{~B}}$, соответственно

$$
\begin{aligned}
& P_{\mathrm{A}}(C)=\frac{(1-C) r_{o \mathrm{~A}}}{(1-C) r_{o \mathrm{~A}}+C r_{o \mathrm{~B}}}, \\
& P_{B}(C)=\frac{C r_{o \mathrm{~B}}}{(1-C) r_{o \mathrm{~A}}+C r_{o \mathrm{~B}}},
\end{aligned}
$$

Для среднего значения какого-либо из четыpex $H$-параметра парного межатомного потенциала Ми-Леннард-Джонса (1) данного сплава было получено выражение [13]:

$$
H\left(\mathrm{~A}_{1-C} \mathrm{~B}_{C}\right)=P_{\mathrm{A}}^{2} H_{\mathrm{A}}+P_{\mathrm{B}}^{2} H_{\mathrm{B}}+2 P_{\mathrm{A}} P_{\mathrm{B}} H_{\mathrm{AB}},
$$

где $H_{\mathrm{AB}}$ - параметр межатомного потенциала (1) для решетки, в которой с равной вероятностью обнаруживаются атомы обеих сортов $\left(P_{i}=0.5\right)$, с учетом релаксации такой решетки к минимуму термодинамического потенциала [13]:

$$
\begin{gathered}
r_{o \mathrm{AB}}=\left(\frac{r_{o \mathrm{~A}}+C_{N} r_{o \mathrm{~B}}^{3}}{1+C_{N}}\right)^{1 / 3}, \\
D_{\mathrm{AB}}=\left(D_{\mathrm{A}} D_{\mathrm{B}}\right)^{1 / 2}, \quad b_{\mathrm{AB}}=\left(b_{\mathrm{A}}+b_{\mathrm{B}}\right) / 2, \\
a_{\mathrm{AB}}=\left(a_{\mathrm{A}}+a_{\mathrm{B}}\right) / 2 .
\end{gathered}
$$

Здесь параметр $C_{N}$, учитывающий разницу в модулях сжатия $\left(B_{i}\right)$ кристаллов чистых компонент для смешанной решетки, в которой с равной вероятностью можно обнаружить атомы обеих сортов, имеет вид

$$
C_{N}=\frac{B_{\mathrm{B}}}{B_{\mathrm{A}}}\left(\frac{r_{o \mathrm{~A}}}{r_{o \mathrm{~B}}}\right)^{3} .
$$

Таким образом, в методе „среднего атома“ твердый раствор $\mathrm{A}_{1-C} \mathrm{~B}_{C}$ двух изоструктурных кристаллов, моделируется виртуальным кристаллом, в котором масса „среднего атома“ и параметры потенциала (1), зависят от концентрации и параметров потенциала (1) для чистых однокомпонентных кристаллов. Данный метод был использован для расчета свойств твердого раствора замещения $\mathrm{Si-Ge}$ в [19] и показал хорошие результаты. 


\section{5. Расчеты свойств макрокристалла}

Применим вышеописанный аналитический метод для изучения свойств неупорядоченного твердого раствора замещения $\mathrm{Au}_{1-C} \mathrm{Fe}_{C}$ с ГЦК-структурой: $k_{n}(\infty)=12$, $k_{p}=0.7405$. Для расчета параметров потенциала сплава ГЦК- $\mathrm{Au}_{1-C} \mathrm{Fe}_{C}$ были использованы следующие значения модулей упругости (при $P=0$ и $T=0 \mathrm{~K}$ ) чистых ГЦК кристаллов: $B_{T}(\mathrm{fcc}-\mathrm{Au})=167 \mathrm{GPa} \quad[20]$; $B_{T}(\mathrm{fcc}-\mathrm{Fe})=88.9 \pm 5.1 \mathrm{GPa}[21]$.

Тогда получим следующие необходимые для расчетов значения параметров:

$$
\begin{gathered}
C_{N}=0.767957, \quad r_{o \mathrm{AuFe}}=2.74124 \cdot 10^{-10} \mathrm{~m}, \\
D_{\mathrm{AuFe}} / k_{B}=\left(D_{\mathrm{Au}} D_{\mathrm{Fe}}\right)^{1 / 2} / k_{B}=7887.06 \mathrm{~K}, \\
b_{\mathrm{AuFe}}=\left(b_{\mathrm{Au}}+b_{\mathrm{Fe}}\right) / 2=12.155, \\
a_{\mathrm{AuFe}}=\left(a_{\mathrm{Au}}+a_{\mathrm{Fe}}\right) / 2=2.460 .
\end{gathered}
$$

Параметры потенциала (1) для чистых металлов из $[18,22]$ и параметры, рассчитанные для сплава эквиатомного состава ГЦК-А $\mathrm{Au}_{0.5} \mathrm{Fe}_{0.5}$ представлены в табл. 1.

Заметим, что в $[5,6]$ значения $c$ для чистых кристаллов были измерены при $300 \mathrm{~K}$ и они равны

$$
\begin{gathered}
c(\mathrm{Au}) /\left[10^{-10} \mathrm{~m}\right]=2.88358-2.88415[5] \\
2.87743-2.88401[6] \\
c(\mathrm{Fe}) /\left[10^{-10} \mathrm{~m}\right]=2.55407-2.57868[5] .
\end{gathered}
$$

Поэтому значения из $[5,6]$ несколько выше, чем соответствующие значения параметра потенциала $r_{o}$, который соответствует расстоянию между центрами ближайших атомов при $P=0$ и $T=0 \mathrm{~K}$.

На рис. 1 и 2 показаны рассчитанные зависимости параметра потенциала $r_{o}$ и температуры Дебая $\Theta$ (при $R=1)$ сплава от $C-$ концентрации $\gamma$-Fe. На рис. 1 сплошная кривая - рассчитанная зависимость для параметра $r_{o}$. Лежащая ниже пунктирная прямая - расчет для $r_{o}(C)$ по формуле Вегарда. Символами показаны экспериментальные данные для $c-$ расстояния между центрами ближайших атомов $\left(c=2^{-1 / 2} l=0.7071 l\right.$, где $l$ - параметр решетки), полученные при $T=300 \mathrm{~K}$ и $P=0$ : сплошные кружки - данные из [5], открытые кружки - из [6].

С помощью (2), (3) и (8) были рассчитаны температуры Дебая и параметры Грюнайзена сплава: $\Theta(m), \gamma(m)$, $q(m), z(m)$, и парциальные значения данных параметров

Таблица 1. Масса атома и параметры потенциала (1) для ГЦК кристаллов $\mathrm{Au}$ и $\mathrm{Fe}$, и для сплава $\mathrm{Au}_{0.5} \mathrm{Fe}_{0.5}$

\begin{tabular}{l|r|c|c|c|c}
\hline \multicolumn{1}{c|}{ Кристалл } & $m$, а.m.u. & $r_{o}, 10^{-10} \mathrm{~m}$ & $D / k_{B}, \mathrm{~K}$ & \multicolumn{1}{c|}{$b$} & $a$ \\
\hline FCC-Au & 196.967 & $2.8751[18]$ & 7419.160 & 16.05 & 2.80 \\
FCC- $\gamma-\mathrm{Fe}$ & 55.847 & $2.5445[22]$ & 8384.467 & 8.26 & 2.12 \\
FCC-Au $_{0.5} \mathrm{Fe}_{0.5}$ & 90.088 & 2.73554 & 7865.022 & 12.39 & 2.48
\end{tabular}

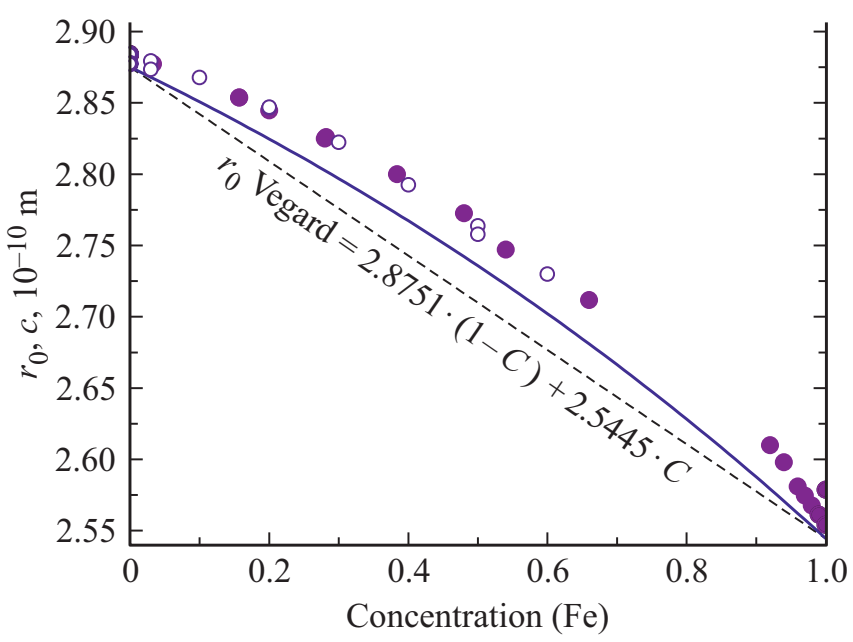

Рис. 1. Изменение кратчайшего расстояния между центрами ближайших атомов в твердом растворе $\mathrm{Au}-\mathrm{Fe}$ в зависимости от концентрации ГЦК- $\gamma$-Fe. Символами показаны экспериментальные результаты из $[5,6]$.

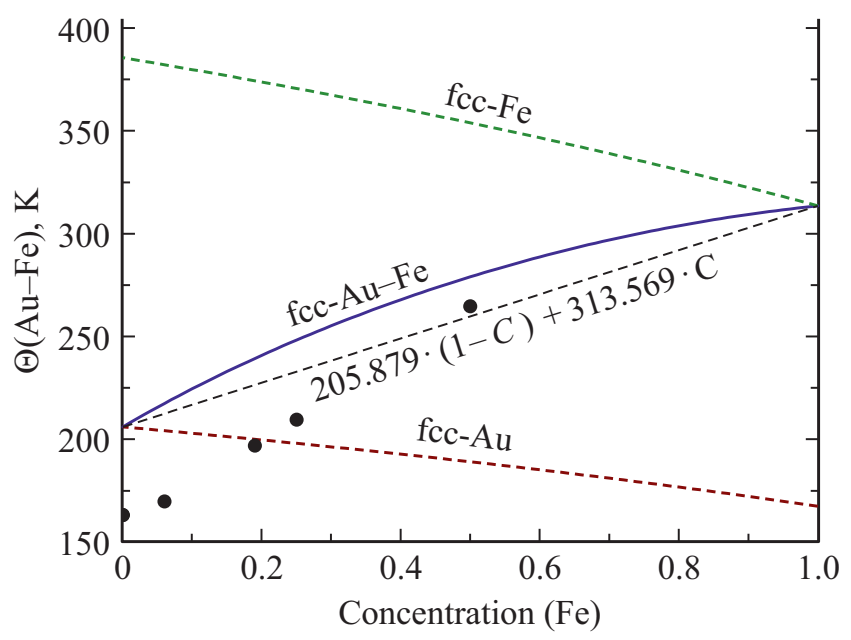

Рис. 2. Концентрационные зависимости для $\Theta(R=1)$ сплава $\mathrm{Au}-\mathrm{Fe}$ (центральная кривая) и для подрешеток ГЦК-Fе (верхняя линия) и ГЦК-Аи (нижняя линия). Точками показаны результаты расчетов из [7].

для составляющий сплав компонентов: $\Theta\left(m_{i}\right), \gamma\left(m_{i}\right)$, $q\left(m_{i}\right), z\left(m_{i}\right)$, т.е. для подрешетки атомов массой $m_{i}$, в которой все параметры межатомного потенциала меняются с концентрацией по формулам (24)-(27). Поэтому на рис. 2 представлены концентрационные зависимости как для температуры Дебая всего сплава ГЦК-Au-Fe (центральная кривая), так и для парциальных температур Дебая подрешеток ГЦК-Fе (верхняя линия) и ГЦК-Аu (нижняя линия). Пунктирной прямой показана зависимость среднего арифметического по концентрации ГЦК- $\gamma$-Fe от температур Дебая чистых кристаллов. Точками на рис. 2 представлены результаты расчетов из [7] для сплава $\mathrm{Au}-\mathrm{Fe}$, которые получены методом функционала плотности (density functional theory). 


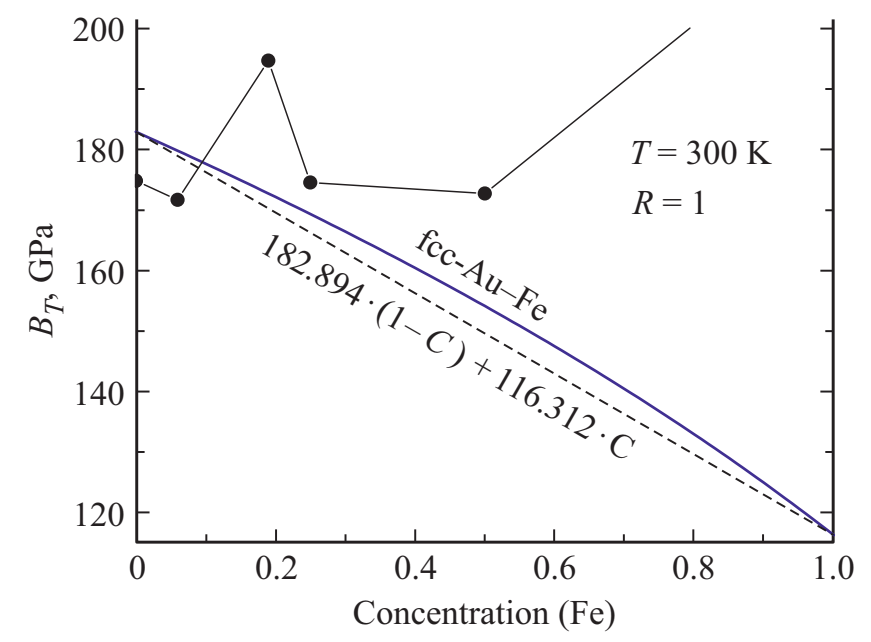

Рис. 3. Концентрационная зависимость для модуля упругости сплава ГЦК-Аu-Fe при $R=1$ и $T=300 \mathrm{~K}$. Точками показаны результаты расчетов из [7].

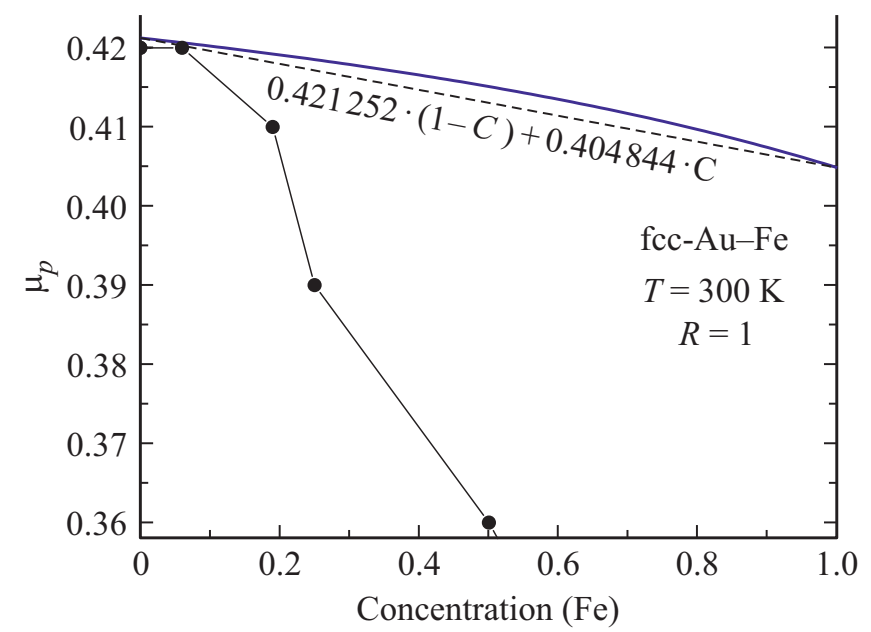

Рис. 4. Концентрационная зависимость для коэффициента Пуассона сплава $\mathrm{Au}-\mathrm{Fe}$ при $R=1$ и $T=300 \mathrm{~K}$. Точками показаны результаты расчетов из[7].

На рис. 3 показана концентрационная зависимость для модуля упругости сплава ГЦК-Au-Fe полученная при $R=1$ и $T=300 \mathrm{~K}$. Пунктирной прямой показана зависимость среднего арифметического по концентрации $\gamma$-Fe от модуля упругости чистых кристаллов. Точками представлены результаты расчетов из [7], которые получены методом функционала плотности (density functional theory).

На рис. 4 показана концентрационная зависимость для коэффициента Пуассона сплава $\mathrm{Au}-\mathrm{Fe}$ при $R=1$ и $T=300 \mathrm{~K}$. Точками показаны результаты расчетов из [7]. Пунктирной прямой показана зависимость среднего арифметического по концентрации $\gamma$-Fe от коэффициента Пуассона чистых кристаллов.

Отметим, что расхождение между нашими результатами и результатами работы [7] обусловлено тем, что для чистого железа мы использовали параметры межатомного потенциала для ГЦК- $\gamma$-Fe из работы [22], а в [7] для параметризации модели сплава ГЦК-Au ${ }_{1-C} \mathrm{Fe}_{C}$ использовались данные для низкотемпературной объемно центрированной кубической структуры железа, т.е. для ОЦК- $\alpha$-Fe. Поэтому на правой вертикальной шкале рис. 1-4 у нас получились данные для ГЦК- $\gamma$-Fe, а в работе [7] - для ОЦК- $\alpha$-Fe. Между тем значения модуля упругости и температуры Дебая для данных фаз при $P=0$ существенно отличаются [21-24]. Например [23]:

$$
\begin{gathered}
B_{T} / \mathrm{GPa}=164(\alpha-\mathrm{Fe}), \quad 146.2(\gamma-\mathrm{Fe}) \\
\Theta / \mathrm{K}=303(\alpha-\mathrm{Fe}), \quad 222.5(\gamma-\mathrm{Fe})
\end{gathered}
$$

Свойства ОЦК- $\alpha$-Fe как для макро-, так и для наножелеза при различных $P-T$-условиях были подробно изучены нами в [24]. В табл. 2 представлены свойства (они указаны в первом столбце) макрокристаллов $(N=\infty)$ FCC-Au, FCC-Fe и $\mathrm{FCC}-\mathrm{Au}_{0.5} \mathrm{Fe}_{0.5}$, рассчитанные при $T=300 \mathrm{~K}$ для изохорического (при $R=1$ ) и изобарического (при $P=0$ ) процессов. Для сравнения можно привести следующие экспериментальные данные этих кристаллов:

для ГЦК-Аu

$$
\begin{gathered}
\Theta /[\mathrm{K}]=165-170[25] ; \quad \gamma=2.95-3.215[25] ; \\
B_{T} /[\mathrm{GPa}]=167.5-180.5[25] ; \quad B^{\prime}(P)=9.58-0.08[25] ; \\
\alpha_{p} /\left[10^{-6} \mathrm{~K}^{-1}\right]=42-42.8[25] ; \\
\sigma(100) /\left[10^{-3} \mathrm{~J} / \mathrm{m}^{2}\right]=1175-1850[26], 1500-1510[27] ; \\
\mu_{p}=0.42[7] ;
\end{gathered}
$$

для ГЦК-Fe

$$
\begin{gathered}
\Theta /[\mathrm{K}]=222.5[23] ; \quad \gamma=2.203[23], \quad 1.3-2.2[28] ; \\
B_{T} /[\mathrm{GPa}]=88.9 \pm 5.1[21], \quad 146.2[23] \\
B^{\prime}(P)=8.9 \pm 0.7[21], \quad 4.67[23] \\
\sigma(100) /\left[10^{-3} \mathrm{~J} / \mathrm{m}^{2}\right]=(1950-2700)[26], \quad 2265[29]
\end{gathered}
$$

Из сравнения видно, что результаты из табл. 2 лучше согласуются с экспериментальными данными, чем результаты, полученные с помощью различных компьютерных моделей в [8].

\section{6. Изменение свойств сплава при переходе к нанокристаллу}

Используя формализм из (2)-(21), массу „среднего атома“ и параметры межатомного потенциала (1) из табл. 1, были рассчитаны свойства сплава эквиатомного состава ГЦК- $\mathrm{Au}_{0.5} \mathrm{Fe}_{0.5}$ как в макро-, так и в нанокристаллических состояниях. При этом, чтобы не нарушались аксиомы равновесной термодинамики, мы полагали, что компоненты сплава распределены по объему равномерно и градиентов плотности либо концентрации в данном кристалле нет. 
Таблица 2. Свойства макрокристаллов ГЦК-Аu, ГЦК-Fе и ГЦК- $\mathrm{Au}_{0.5} \mathrm{Fe}_{0.5}$, рассчитанные при $T=300 \mathrm{~K}$ для изохорического и изобарического процессов

\begin{tabular}{|c|c|c|c|c|c|c|}
\hline Кристалл & \multicolumn{2}{|c|}{ FCC-Au } & \multicolumn{2}{|c|}{$\mathrm{FCC}-\gamma-\mathrm{Fe}$} & \multicolumn{2}{|c|}{ FCC- $\mathrm{Au}_{0.5} \mathrm{Fe}_{0.5}$} \\
\hline $\begin{array}{c}\text { Свойство } \\
\text { [единица измерения] }\end{array}$ & $\begin{array}{c}c / r_{o}=1 \\
P=2.27 \mathrm{GPa}\end{array}$ & $\begin{array}{c}P=0 \\
c / r_{o}=1.0043\end{array}$ & $\begin{array}{c}c / r_{o}=1 \\
P=1.91 \mathrm{GPa}\end{array}$ & $\begin{array}{c}P=0 \\
c / r_{o}=1.0057\end{array}$ & $\begin{array}{c}c / r_{o}=1 \\
P=2.14 \mathrm{GPa}\end{array}$ & $\begin{array}{c}P=0 \\
c / r_{o}=1.0048\end{array}$ \\
\hline$\Theta$ - температура Дебая $[\mathrm{K}]$ & 205.879 & 198.043 & 313.569 & 304.650 & 278.924 & 269.508 \\
\hline$\gamma=-(\partial \ln \Theta / \partial \ln v)_{T}$ & 3.0005 & 3.0008 & 1.7040 & 1.7042 & 2.3903 & 2.3906 \\
\hline$q \cdot 10^{3}=(\partial \ln \gamma / \partial \ln v)_{T} \cdot 10^{3}$ & 7.8671 & 7.5662 & 6.0378 & 5.8649 & 8.0271 & 7.7544 \\
\hline$z=-(\partial \ln q / \partial \ln v)_{T}$ & 3.0161 & 3.0158 & 1.7160 & 1.7158 & 2.4063 & 2.4060 \\
\hline$B_{T}=-v(\partial P / \partial v)_{T}[\mathrm{GPa}]$ & 185.162 & 166.379 & 118.222 & 107.819 & 156.188 & 141.368 \\
\hline$B^{\prime}(P)=\left(\partial B_{T} / \partial P\right)_{T}^{1)}$ & 8.194 & 8.350 & 5.388 & 5.494 & 6.875 & 7.007 \\
\hline$\alpha_{p}=\gamma C_{v} /\left(B_{T} V\right)\left[10^{-6} \mathrm{~K}^{-1}\right]$ & 39.071 & 42.997 & 48.704 & 52.662 & 42.062 & 45.936 \\
\hline$\alpha_{p} \cdot B_{T}=(\partial P / \partial T)_{\nu}\left[10^{-3} \mathrm{GPa} / \mathrm{K}\right]$ & 7.2344 & 7.1539 & 5.7579 & 5.6779 & 6.5696 & 6.4939 \\
\hline$C_{v} /\left(N k_{B}\right)$ & 2.9346 & 2.9395 & 2.8510 & 2.8591 & 2.8813 & 2.8890 \\
\hline$C_{p} /\left(N k_{B}\right)=\left[C_{v} /\left(N k_{B}\right)\right]\left(1+\gamma \alpha_{p} T\right)$ & 3.0379 & 3.0532 & 2.9220 & 2.9361 & 2.9683 & 2.9842 \\
\hline$\Theta^{\prime}(P)=(\partial \Theta / \partial P)_{T}[\mathrm{~K} / \mathrm{GPa}]^{1)}$ & 3.3376 & 3.5708 & 4.5195 & 4.8160 & 4.2673 & 4.5589 \\
\hline$C_{v}^{\prime}(P) /\left(N k_{B}\right)\left[10^{-3} / \mathrm{GPa}\right]^{1)}$ & -2.1026 & -2.1343 & -4.1671 & -4.3357 & -3.5356 & -3.6708 \\
\hline$\alpha_{p}^{\prime}(P)\left[10^{-6} /(\mathrm{K} \mathrm{GPa})\right]^{1)}$ & -1.5469 & -1.9318 & -1.8806 & -2.2768 & -1.6353 & -2.0116 \\
\hline$C_{p}^{\prime}(P) /\left(N k_{B}\right)\left[10^{-3} / \mathrm{GPa}\right]^{1)}$ & -6.2671 & -7.3343 & -7.0152 & -7.7846 & -7.0257 & -7.9641 \\
\hline$\sigma(100)\left[10^{-3} \mathrm{~J} / \mathrm{m}^{2}\right]$ & 1545.26 & 1531.38 & 2231.69 & 2206.08 & 1810.29 & 1792.46 \\
\hline$X_{s c} \cdot 10^{3}=10^{3} \cdot \sigma /\left(c \cdot B_{T}\right)$ & 29.027 & 31.876 & 74.188 & 79.960 & 42.370 & 46.129 \\
\hline$\mu_{p}$ - коэффициент Пуассона & 0.4203 & 0.4274 & 0.4033 & 0.4103 & 0.4139 & 0.4210 \\
\hline$\sigma^{\prime}(T)_{v}\left[10^{-6} \mathrm{~J} /\left(\mathrm{m}^{2} \mathrm{~K}\right)\right]$ & -51.33 & -50.98 & -63.61 & -63.08 & -55.63 & -55.26 \\
\hline$\sigma^{\prime}(T)_{P}\left[10^{6} \mathrm{~J} /\left(\mathrm{m}^{2} \mathrm{~K}\right)\right]$ & -91.51 & -98.927 & -135.90 & -144.12 & -106.26 & -113.95 \\
\hline$\sigma^{\prime}(P)_{T}\left[10^{-3} \mathrm{~J} /\left(\mathrm{m}^{2} \mathrm{GPa}\right)\right]^{1)}$ & 5.551 & 3.571 & 12.554 & 14.270 & 7.704 & 9.035 \\
\hline
\end{tabular}

Пр имеч ан и е. ${ }^{1)}$ - Рассчитано численным дифференцированием параметра по давлению вдоль изотермы.

На рис. 5-14 показаны изоморфно-изомерноизотермические (т.е. при $f=1 ; N=\infty, 306 ; T=100$, $300,500 \mathrm{~K})$ зависимости для различных свойств сплава ГЦК-Аu $\mathrm{Au}_{0.5} \mathrm{Fe}_{0.5}$. Толстые линии $1,3,5$ изотермы $T=500,300,100 \mathrm{~K}$ для макрокристалла $(N=\infty)$. Тонкие линии $2,4,6-$ изотермы $T=500$, $300,100 \mathrm{~K}$ для нанокристалла кубической формы из $N=f N_{p o}^{3} / \alpha=306$ атомов (т.е. $N_{p o}=6, f=1$, $\left.k_{n}^{*}=0.8822\right)$.

На рис. 5 показано изменение изотерм уравнения состояния (т.е. зависимости $P$ (в $\mathrm{GPa})$ от нормированного объема $\left.v / v_{o}=\left(c / r_{o}\right)^{3}=R^{-3}\right)$ при переходе от макрокристалла к нанокристаллу при различных температурах. Из рис. 5 видно, что при определенном значении относительного объема $\left(v / v_{o}\right)_{0}$ изотермические зависимости $P\left(v / v_{o}\right)$ для нанокристалла и макрокристалла пересекаются. Таким образом, при $\left(v / v_{o}\right)_{0}$ поверхностное давление становится равным нулю: $P_{s f}\left(v / v_{o}\right)_{0}=P($ Macro $)-P($ Nano $)=0$, следовательно: $\Delta p\left(v / v_{o}\right)_{0}=1$. При $v / v_{o}<\left(v / v_{o}\right)_{0}$ поверхностное давление сжимает нанокристалл $\left(P_{s f}>0\right)$, а при $v / v_{o}>\left(v / v_{o}\right)_{0}$ поверхностное давление нанокристалл растягивает: $P_{s f}<0$. Расчеты показали, что величина $\left(v / v_{o}\right)_{0}$ уменьшается как при изоморфно-изомерном $(f, N$ - const) росте температуры, так и при изоморфно-изотермическом ( $f, T$ - const) уменьшении $N$, или при изомерно-изотермическом $(N, T-$

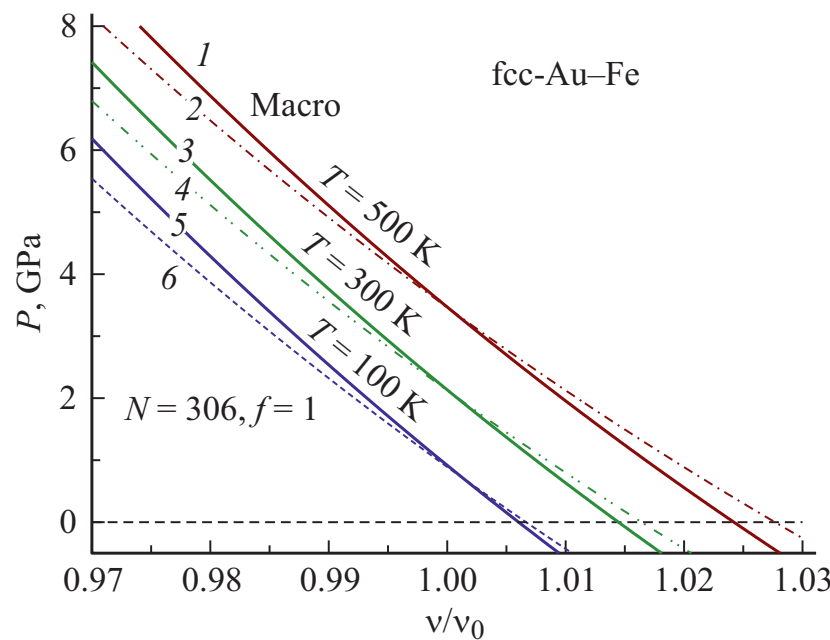

Рис. 5. Изменение уравнения состояние ГЦК- $\mathrm{Au}_{0.5} \mathrm{Fe}_{0.5}$ при различных температурах и числе атомов. Сплошные линии 1,3,5 получены для макрокристалла, а линии 2,4,6 - для нанокристалла из $N=306$ атомов.

const) отклонении формы нанокристалла от наиболее энергетически оптимальной формы (для RP-модели это куб).

Из рис. 5 также следует, что давление в нанокристалле переходит через ноль при значение $v / v_{o}$ большем, 


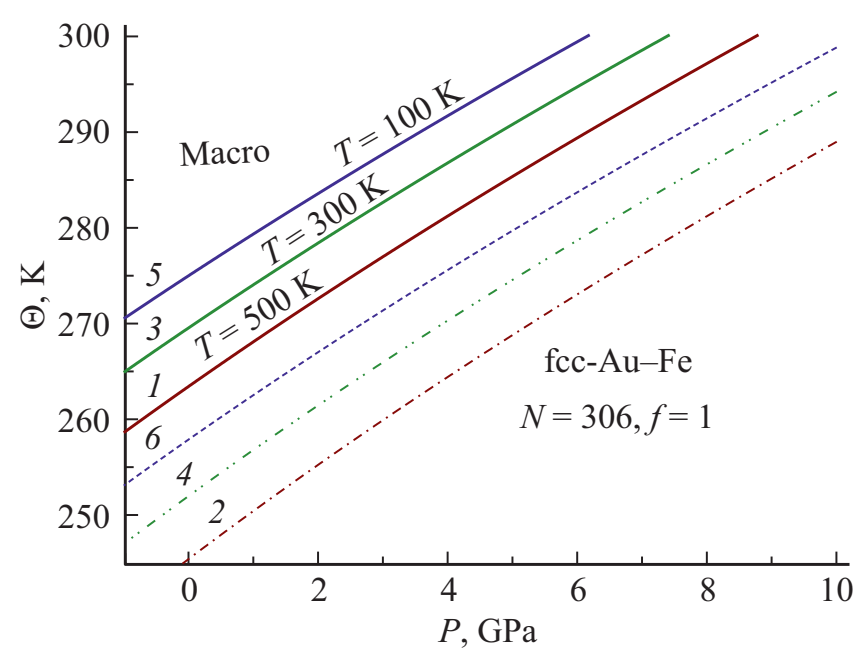

Рис. 6. Барическая зависимость температуры Дебая для сплава ГЦК- $\mathrm{Au}_{0.5} \mathrm{Fe}_{0.5}$ при различных температурах для макрои нанокристалла.

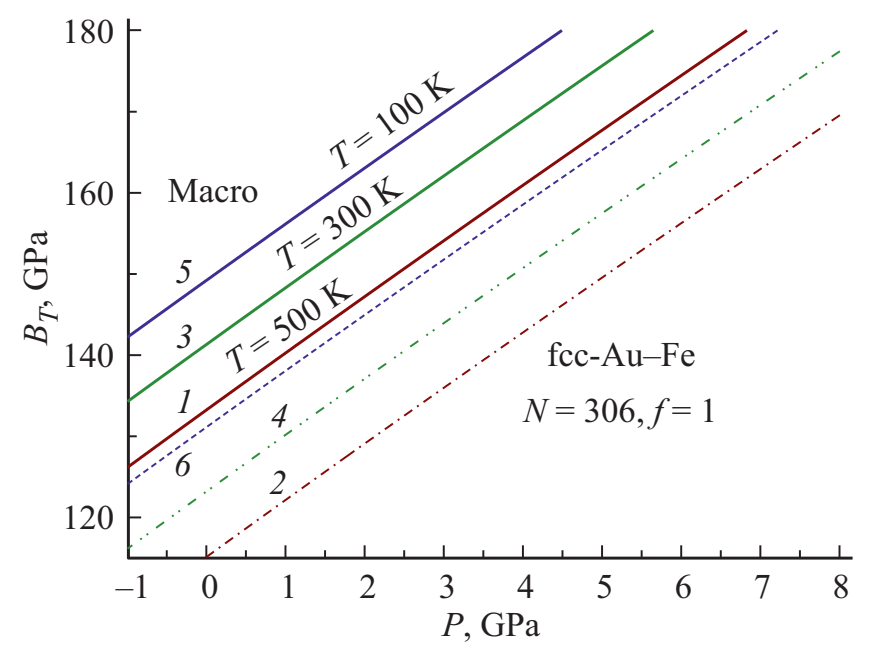

Рис. 7. Изменение модуля упругости для сплава ГЦК$\mathrm{Au}_{0.5} \mathrm{Fe}_{0.5}$ с ростом давления при различных температурах для макро- и нанокристалла.

чем для макрокристалла. Т.е. при изобарическом (при $P($ Macro $)=P(N=306, f=1)=0)$ уменьшении числа атомов нанокристалл со свободной поверхностью Гиббса растянут отрицательным поверхностным давлением тем больше, чем выше температура. Поэтому полагать, что при изобарическом уменьшении числа атомов плотность нанокристалла сохраняется некорректно. При этом, чем дальше от точки нулевого поверхностного давления лежит значение давления изобары, тем больше будет меняться плотность нанокристалла при изотермоизоморфном уменьшении в нем числа атомов.

Рассчитав зависимость $P\left(f, N, T ; v / v_{o}\right)$ и зависимость какого-либо свойства $X\left(f, N, T ; v / v_{o}\right)$ вдоль определенной изотермы при заданных значениях $f=1 ; N=\infty$, 306 , можно получить барическую зависимость этого свойства $X(P)$ вдоль этой же изотермы. На рис. 6-14 показаны полученные таким путем барические зависимости различных свойств однородного твердого раствора замещения ГЦК- $\mathrm{Au}_{0.5} \mathrm{Fe}_{0.5}$.

На рис. 6 показаны барические зависимости для температуры Дебая $\Theta$ при различных температурах, как для макрокристалла (сплошные линии $1,3,5$ ), так и для нанокристалла (линии $2,4,6$ ). Как видно из рис. 6 , величина $\Theta$ уменьшается как при изомерно-изобарическом увеличении температуры, так и изотермо-изобарическом уменьшении числа атомов в нанокристалле.

На рис. 7 показаны барические зависимости для $B_{T}=-v(\partial P / \partial v)_{T}$ - модуля упругости (в GPa) при различных температурах, как для макрокристалла (сплошные линии 1,3,5), так и для нанокристалла (линии $2,4,6)$. Как видно из рис. 7 величина $B_{T}$ уменьшается как при изомерно-изобарическом увеличении температуры, так и при изотермо-изобарическом уменьшении числа атомов в нанокристалле.

На рис. 8 показаны барические зависимости для $B^{\prime}(P)=\left(\partial B_{T} / \partial P\right)_{T}-$ производной модуля упругости по давлению вдоль изотермы. Как видно из рис. 8 величина $B^{\prime}(P)$ увеличивается при изомерно-изобарическом увеличении температуры. При этом на изотермах при определенном давлении $\left(P_{B}\right)$ имеются точки, в которых барические зависимости $B^{\prime}(P)$ для макро- и нанокристалла пересекаются: $B^{\prime}(P)_{\infty}-B^{\prime}(P)_{N}=0$. Легко понять, что в этих точках пересечения размерная зависимость функции $B^{\prime}(P)$ меняет знак. При $P<P_{B}$ величина $B^{\prime}(P)$ растет при изотермо-изобарном уменьшении $N$, а при $P>P_{B}$ величина $B^{\prime}(P)$ уменьшается.

На рис. 9 показаны барические зависимости для $\alpha_{p}=[\partial \ln (V) / \partial T]_{P}-$ коэффициента теплового расширения $\left(\right.$ в $\left.10^{-6} 1 / \mathrm{K}\right)$ при $T=100,300,500 \mathrm{~K}$, как для макро(линии $1,3,5$ ), так и для нано-кристалла (линии $2,4,6$ ).

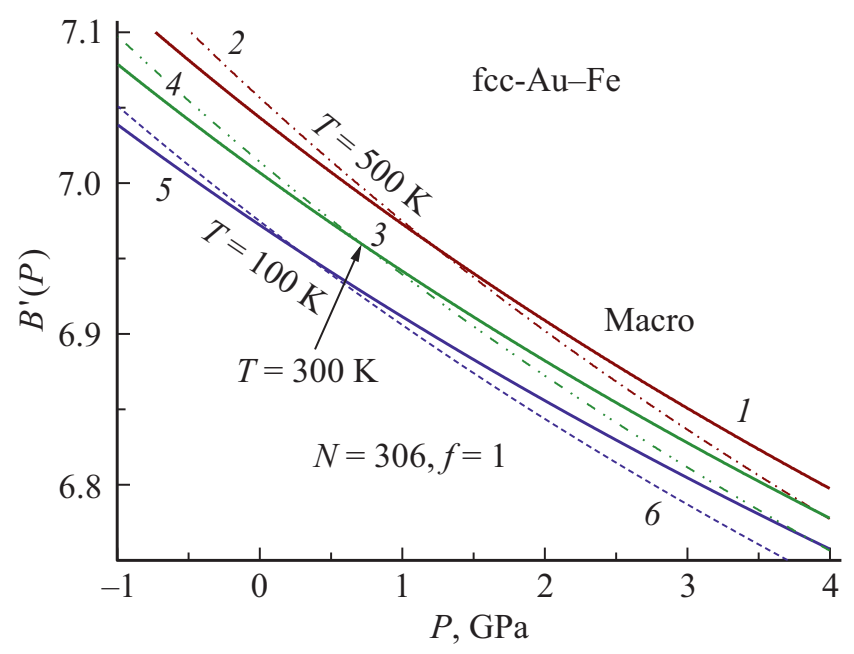

Рис. 8. Барическая зависимость производной модуля упругости по давлению для ГЦК- $\mathrm{Au}_{0.5} \mathrm{Fe}_{0.5}$ при различных температурах для макро- и нанокристалла. 


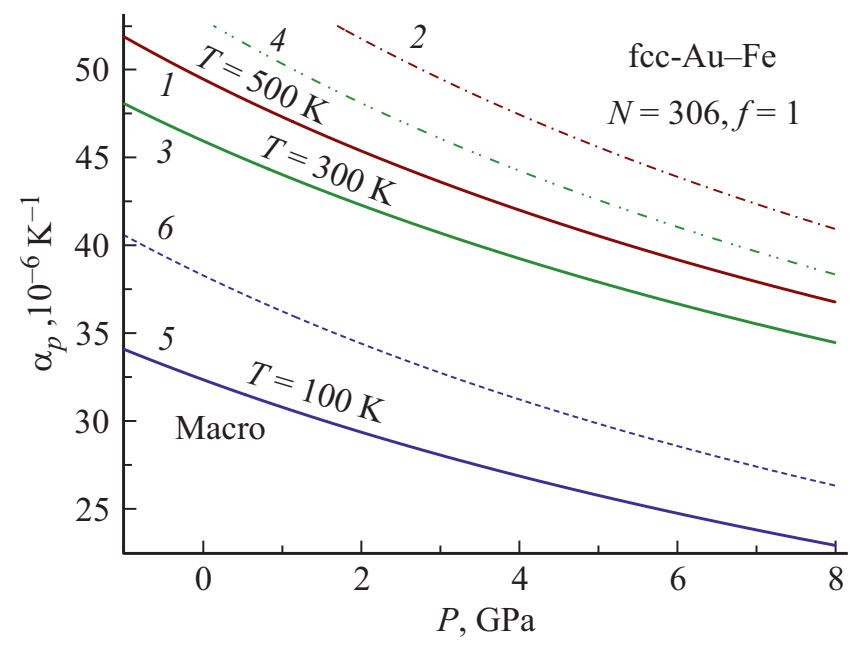

Рис. 9. Барическая зависимость коэффициента теплового расширения для сплава ГЦК- $\mathrm{Au}_{0.5} \mathrm{Fe}_{0.5}$ для макро- и нанокристалла.

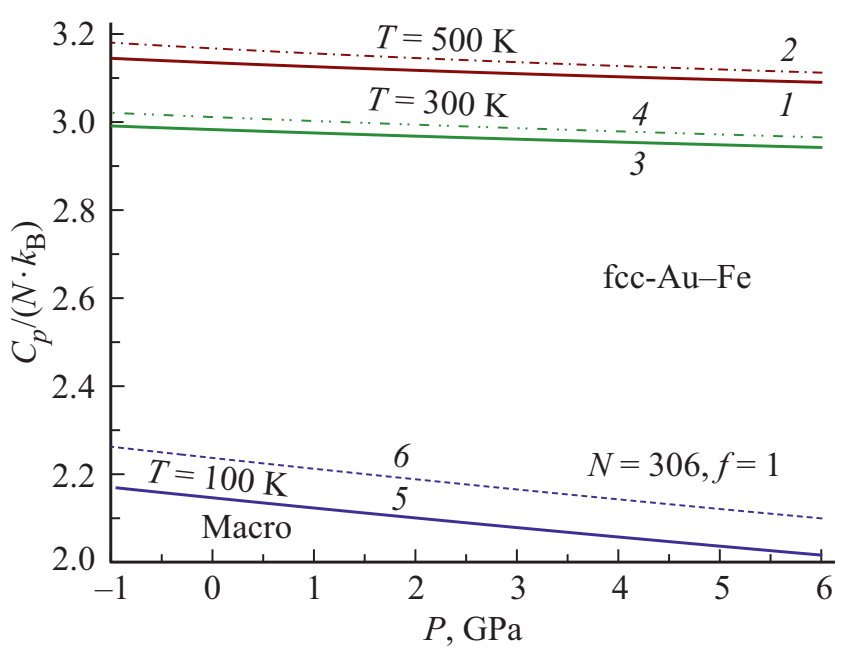

Рис. 10. Барическая зависимость изобарной теплоемкости для $\mathrm{Au}_{0.5} \mathrm{Fe}_{0.5}$.

Из рис. 9 видно, что для нанокристалла величина $\alpha_{p}$ всегда больше, чем для макрокристалла.

На рис. 10 показаны барические зависимости для $C_{p}=C_{\nu}\left(1+\gamma \cdot \alpha_{p} \cdot T\right)$ - изобарной теплоемкости сплава ГЦК- $\mathrm{Au}_{0.5} \mathrm{Fe}_{0.5}$ при различных температурах для макро- и нанокристалла. Из рис. 10 видно, что размерная зависимость функции $C_{p}(N)$ наиболее заметна при низких температурах и давлениях. Причины такого поведения функции $C_{p}(N)$ были подробно изучены в [11].

На рис. 11 показаны барические зависимости для $\sigma-$ удельной поверхностной энергии (в $10^{-3} \mathrm{~J} / \mathrm{m}^{2}$ ) грани (100) сплава ГЦК-Аu $0.5 \mathrm{Fe}_{0.5}$ при различных температурах. Из рис. 11 видно, что при $P=0$ величина $\sigma$ уменьшается при уменьшении $N$ тем заметнее, чем выше температура. Но при низких температурах и высоких давлениях на изотерме имеются две $P$-точки, где удельная поверхностная энергия не зависит от размера нанокристалла: $\sigma(N)=\sigma(\infty)$. Впервые такие Р-точки были обнаружены нами в [24] при изучении свойств ОЦК-Fе, а потом и для Ge [12]. С ростом температуры эти Р-точки сближаются, и при высоких температуpax таких Р-точек на изотерме уже нет. В области, оконтуренной Р-точками величина $\sigma$ возрастает при изотермо-изобарическом уменьшении размера нанокристалла. Такое поведение функции $\sigma(P, N)$ обусловлено тем, что при низких температурах и высоких давлениях поверхностное давление сжимает нанокристалл, что и приводит к появлению первой Р-точки и к соотношению: $\sigma(N)>\sigma(\infty)$. С ростом давления функция $\sigma$

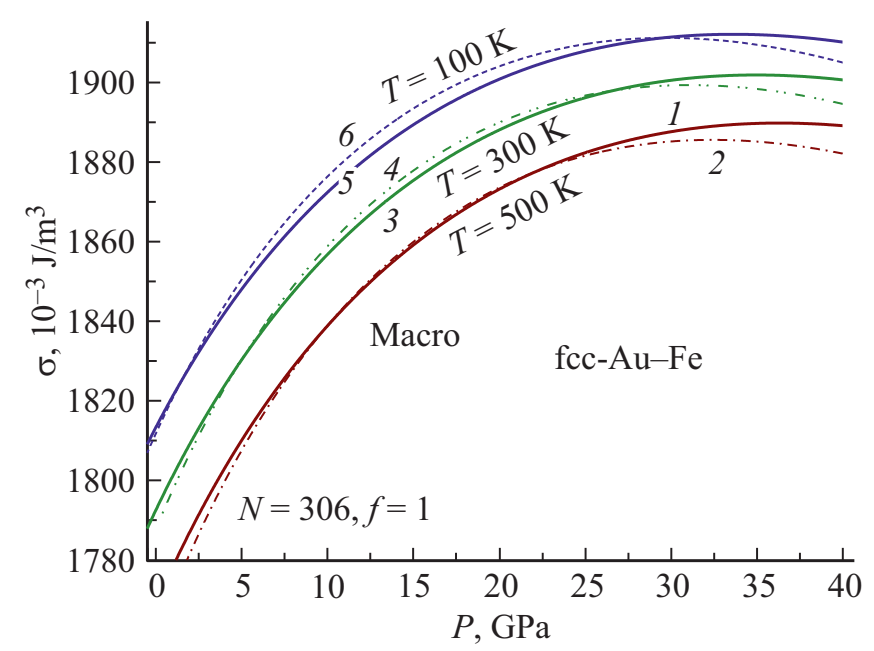

Рис. 11. Барическая зависимость удельной поверхностной энергии грани (100) для ГЦК- $\mathrm{Au}_{0.5} \mathrm{Fe}_{0.5}$ при различных температурах.

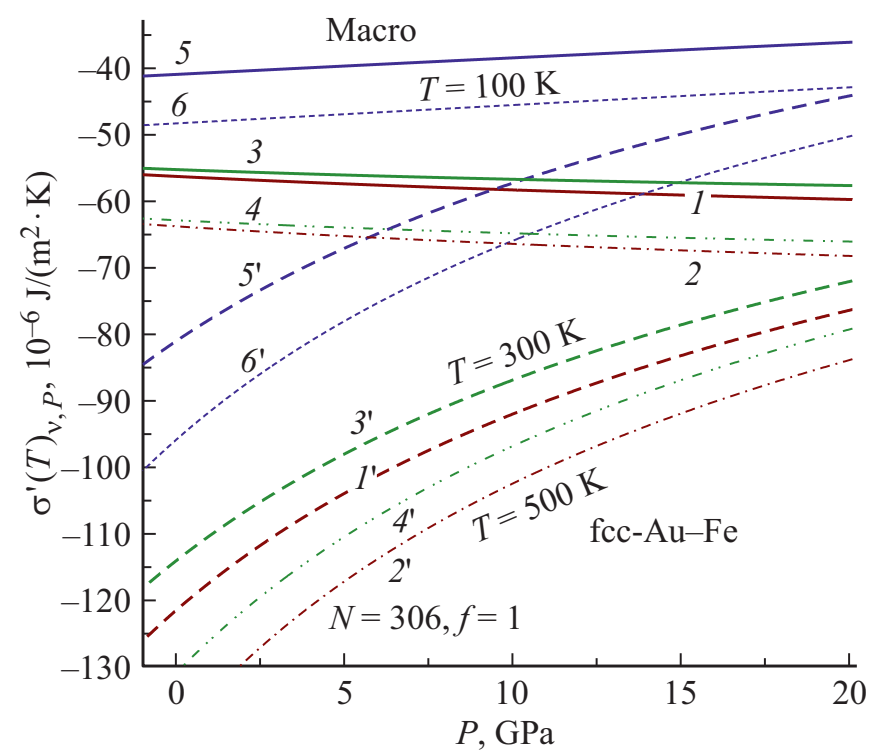

Рис. 12. Барическая зависимость для $\sigma^{\prime}(T)_{v}-$ изохорной (линии $\left.1-6^{\prime}\right)$ и $\sigma^{\prime}(T)_{P}-$ изобарной ( возрастающие кривые $\left.1^{\prime}-6^{\prime}\right)$ производных удельной поверхностной энергии по температуре для ГЦК-Аu ${ }_{0.5} \mathrm{Fe}_{0.5}$. 


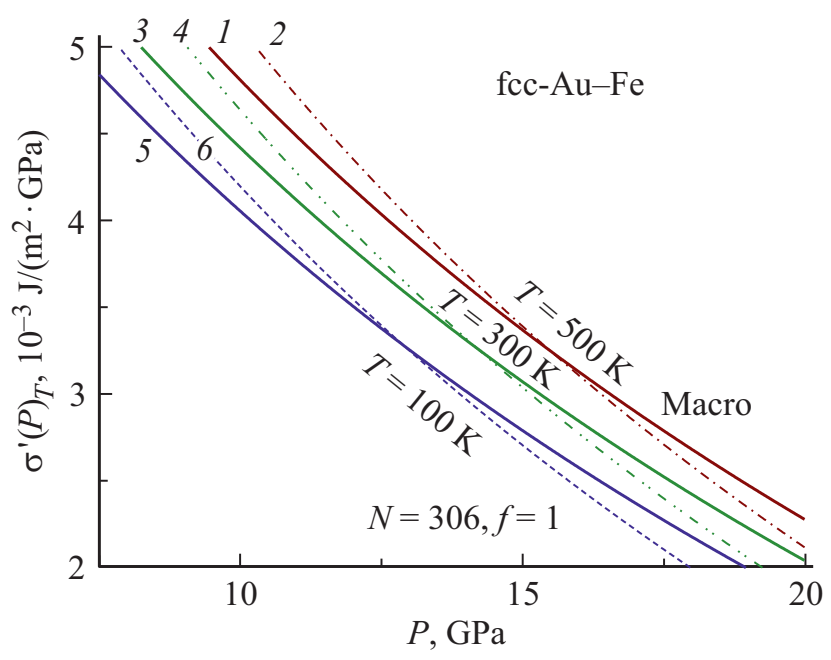

Рис. 13. Барическая зависимость производной удельной поверхностной энергии по давлению для ГЦК- $\mathrm{Au}_{0.5} \mathrm{Fe}_{0.5}$ при различных температурах.

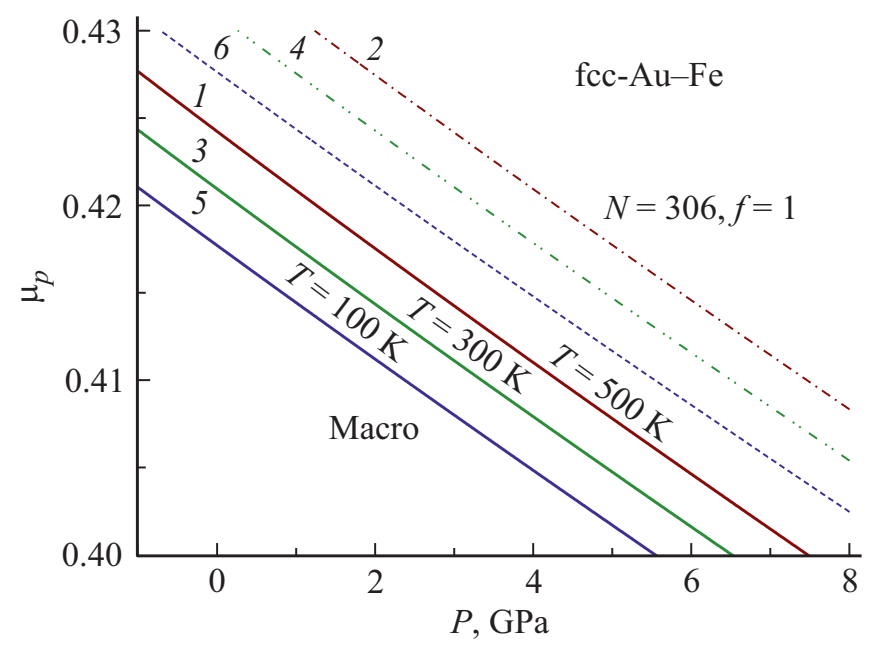

Рис. 14. Барическая зависимость коэффициента Пуассона для сплава ГЦК- $\mathrm{Au}_{0.5} \mathrm{Fe}_{0.5}$ при различных температурах.

для нанокристалла уменьшается заметнее, чем для макрокристалла, что и приводит к образованию второй Р-точки на изотерме. При изобарном росте температуры поверхностное давление уменьшается, что и приводит к исчезновению области с Р-точками.

На рис. 12 показаны барические зависимости для $\sigma^{\prime}(T)_{v}$ - изохорной $(6$ верхних линий $1-6)$ и $\sigma^{\prime}(T)_{P}-$ изобарной (6 нижних возрастающих кривых $\left.1^{\prime}-6^{\prime}\right)$ производных удельной поверхностной энергии по температуре (в $10^{-6} \mathrm{~J} /\left(\mathrm{m}^{2} \mathrm{~K}\right)$ ) для ГЦК- $\mathrm{Au}_{0.5} \mathrm{Fe}_{0.5}$. Как видно из рис. 12, при низких давлениях выполняется $\left|\sigma^{\prime}(T)_{\nu}\right|<\left|\sigma^{\prime}(T)_{P}\right|$. При $P=0$ и $T \ll \Theta$ величина $\sigma^{\prime}(T)_{v}$ практически не зависит от температуры. При переходе к нанокристаллу величины $\sigma^{\prime}(T)_{\nu}|<| \sigma^{\prime}(T)_{P} \mid$ возрастают.
На рис. 13 показаны барические зависимости для $\sigma^{\prime}(P)_{T}=(\partial \sigma / \partial P)_{T}-$ производной удельной поверхностной энергии грани (100) по давлению (в $\left.10^{-3} \mathrm{~J} /\left(\mathrm{m}^{2} \mathrm{GPa}\right)\right)$ для сплава ГЦК-Аu $0.5 \mathrm{Fe}_{0.5}$ при различных температурах. Как видно из рис. 13 величина $\sigma^{\prime}(P)_{T}$ увеличивается при изомерно-изобарическом увеличении температуры. При этом на изотермах при определенном давлении $\left(P_{\sigma}\right)$ имеются точки, в которых барические зависимости $\sigma^{\prime}(P)_{T}$ для макро- и нанокристалла пересекаются: $\sigma^{\prime}(P)_{T \infty}-\sigma^{\prime}(T)_{T N}=0$. Легко понять, что в этих точках пересечения размерная зависимость функции $\sigma^{\prime}(P)_{T}$ меняет знак. При $P<P_{\sigma}$ величина $\sigma^{\prime}(T)_{T}$ растет при изотермо-изобарном уменьшении $N$, а при $P>P_{\sigma}$ величина $\sigma^{\prime}(P)_{T}$ уменьшается. Здесь видна аналогия между барическими зависимостями $\sigma^{\prime}(P)_{T}$ и $B^{\prime}(P)$ из рис. 8.

На рис. 14 показаны барические зависимости для $\mu_{p}$ — коэффициента Пуассона сплава ГЦК- $\mathrm{Au}_{0.5} \mathrm{Fe}_{0.5}$ при различных температурах. Как видно из рис. 14 величина $\mu_{p}$ увеличивается как при изомерно-изобарическом увеличении температуры, так и при изотермо-изобарическом уменьшении числа атомов в нанокристалле.

\section{7. Обсуждение результатов для нанокристалла}

Так как о достоверности наших результатов, полученных для концентрационных зависимостей свойств макрокристалла fcc- $\mathrm{Au}_{1-C} \mathrm{Fe}_{C}$ можно судить по согласию с экспериментальными данными и с результатами других авторов, то для нанокристалла fсc- $\mathrm{Au}_{0.5} \mathrm{Fe}_{0.5}$ экспериментальных данных и расчетов других авторов пока нет. Поэтому мы обсудим качественное согласие, полученных нами для нанокристалла fcc- $\mathrm{Au}_{0.5} \mathrm{Fe}_{0.5}$ размерных зависимостей с известными из литературы размерными зависимостями свойств различных нанокристаллов.

Если размерная зависимость температуры Дебая, коэффициента теплового расширения, теплоемкости и межатомного расстояния при $P=0$ хорошо изучена [30] и споров не вызывает, то размерная зависимость для модуля упругости различных веществ крайне противоречива.

Как ясно следует из рис. 7, модуль упругости $B_{T}(N)$ в рамках нашей модели уменьшается при изотермо-изобарно-изоморфном уменьшении числа атомов $N$. Вместе с тем, в некоторых статьях (например, в [31] для Мо и $\mathrm{Ni}$, в [32] для $\mathrm{Si}$ и $\mathrm{Ge}$, и в [33] для алмаза) обнаружено увеличение модуля упругости $B_{T}$ при уменьшении размера (или числа атомов $N$ ) нанокристалла. По нашему мнению, это связано с методами использованными в указанных работах для экспериментального или теоретического изучения нанокристаллов.

Например, в [31] экспериментально изучался спрессованный нанопорошок Мо и также Ni. Измерения, в соответствии с законом Холла-Петча (Hall-Petch), 
показали увеличение $B_{T}$ спрессованного нанопорошка, как это признали и сами авторы. При этом, для получения величины $B_{T}$ использовалось уравнение состояния Берча-Мурнагана (Birch-Murnaghan), в котором фиксировались два параметра: $v(P=0) / v_{o}=1$ и $B^{\prime}(P)=4$ (cм. [31], Table I), т.е. полагалась их независимость от размера нанокристалла. Но, как следует из полученных нами результатов (рис. 5 и 8), параметры $v(P=0)$ и $B^{\prime}(P)$ увеличиваются при изобарическом $P(N)=0$ уменьшении $N$.

В работе [32] изучение функции $B_{T}(N)$ было проведено квантовым методом Монте-Карло при $T=0 \mathrm{~K}$. При этом уменьшение $N$ проходило при постоянной (или даже при увеличивающейся (см. [32], Table IV) с уменьшением $N$ плотности, что и привело к закономерному росту величины $B_{T}$ с уменьшением $N$.

Обнаруженное в [33] путем изучения рамановского спектра увеличение модуля упругости нано-алмаза, связано с тем же эффектом Холла-Петча (Hall-Petch) для образца из спрессованных 2-5 nm алмазных частиц, которые были получены детонационным путем. Также в увеличение $B_{T}(N)$ могло внести и то, что нано-порошок алмаза был перед прессованием еще и механоактивирован в планетарно-шаровой мельнице. Это несомненно привело к существенному изменению свойств поверхности наногранул алмаза. Сжатие и механоактивация нано-порошка алмаза привели к росту величины $B_{T}$ в приповерхностной области нанокристаллов. С уменьшением размера нанокристалла вклад приповерхностной области растет. Это и привело к увеличению $B_{T}(N)-$ модуля упругости всего нанокристалла при уменьшении $N$. Данное утверждение подтверждается также и тем, что нано-порошок алмаза приготовленный без сжатия и механоактивации в [34] показал уменьшение модуля упругости при уменьшении размера наногранул алмаза.

В данной статье изучается нанокристалл со свободной (и не механоактивированной) поверхностью Гиббса в форме прямоугольного параллелепипеда (RP-модель). B рамках RP-модели было показано [35], что для такого нанокристалла модуль упругости $B_{T}(N)$ уменьшается при изотермо-изоморфном уменьшении числа атомов $N$. Такое же уменьшение функции $B_{T}(N)$ при уменьшении $N$ было получено и другими методами для нанокристалла со свободной поверхностью Гиббса и во многих других работах. Например: для $\mathrm{Cu}, \mathrm{Pd}$ и $\mathrm{Si}$ в [36], для $\mathrm{Cu}$ и алмаза в [37], для $\mathrm{Pb}$ и $\mathrm{Au}$ в [38], для $\mathrm{Si} \mathrm{в}[39,40]$, для $\mathrm{Cu}$ в [41]. В статье [42] в рамках RP-модели на примере аргона было показано, что при изоморфно-изобарическом уменьшении размера нанокристалла функция $B_{T}(N)$ уменьшаются, а в случае изоморфно-изохорического уменьшения размера нанокристалла модуль упругости возрастает.

Таким образом, полученное в разных теоретических или экспериментальных работах различие в зависимости модуля упругости от размера нанокристалла может быть обусловлено как состоянием поверхности, так и характером процесса, при котором происходило изотермоизоморфное уменьшение размера нанокристалла в этих работах.

Ввиду такой неоднозначности с размерной зависимостью модуля упругости $B_{T}(N)$, в литературе очень мало работ по изучению размерной зависимости коэффициента Пуассона $\mu_{p}(N)$. Вместе с тем, имеющиеся экспериментальные [34] и теоретические [41] исследования указывают на увеличение коэффициента Пуассона при переходе от макро- к нанокристаллу. Это согласуется с результатами для функции $\mu_{p}(N)$, полученными в рамках RP-модели и представленными на рис. 14.

Что касается размерной зависимости для производной модуля упругости по давлению и для поверхностных свойств, то существующие экспериментальные либо теоретические методы пока не позволили получить эти зависимости даже для однокомпонентных нанокристаллов. Поэтому в литературе этих зависимостей нет.

\section{8. Заключение}

Исходя из параметров парного межатомного потенциала Ми-Леннард-Джонса для ГЦК-Аu и ГЦК-Fе определены параметры парного межатомного потенциала Ми-Леннард-Джонса для неупорядоченного сплава замещения ГЦК-Au-Fe. На основе данных параметров рассчитаны концентрационные зависимости решеточных свойств для макрокристалла данного сплава. Расчеты 20 свойств макрокристаллов ГЦК-Аu, ГЦК-Fе и ГЦК- $\mathrm{Au}_{0.5} \mathrm{Fe}_{0.5}$ показали хорошее согласие с экспериментальными данными.

На основе RP-модели нанокристалла рассчитано уравнение состояния $P(v, T ; N)$ и барические зависимости как упругих, так и для тепловых и поверхностных свойств сплава ГЦК- $\mathrm{Au}_{0.5} \mathrm{Fe}_{0.5}$. Расчеты были проведены при температурах $T=100,300$ и $500 \mathrm{~K}$, как для макрокристалла $(N=\infty)$, так и для нанокристалла кубической формы из $N=306$ атомов.

Показано, что при изоморфно-изотермо-изобарическом (т.е. при $f$ и $T=\mathrm{const} ; P=0$ ) уменьшении размера нанокристалла его температура Дебая, модуль упругости и удельная поверхностная энергия уменьшаются, а его удельный объем, коэффициент теплового расширения, удельная теплоемкость, $\left|\sigma^{\prime}(T)_{v}\right|, \sigma^{\prime}(T)_{P} \mid$ и коэффициент Пуассона увеличиваются. Причем, эти размерные изменения тем заметнее, чем выше температура, или чем заметнее форма нанокристалла отклонена от энергетически оптимальной формы (для RP-модели это куб).

При низких температурах в определенной области давлений удельная поверхностная энергия возрастает при изоморфно-изотермо-изобарическом уменьшении числа атомов в нанокристалле. С ростом температуры эта область давлений уменышается и исчезает. 


\section{Благодарности}

Автор выражает благодарность С.П. Крамынину, Н.Ш. Газановой и 3.М. Сурхаевой за плодотворные дискуссии и помощь в работе.

\section{Финансирование работы}

Работа выполнена при финансовой поддержке РФФИ (грант № 18-29-11013_мк и Программы Президиума РАН (программа № 6, грант 2-13).

\section{Конфликт интересов}

Автор заявляет, что у него нет конфликта интересов.

\section{Список литературы}

[1] M. Saoudi, H. Fritzsche, G.J. Nieuwenhuys, M.B.S. Hesselberth. Phys.Rev. Lett. 100, 5, 057204 (2008). https://doi.org/10.1103/PhysRevLett.100.057204

[2] Q. Zhang, P. Li, Y. Wen, C. Zhao, J.W. Zhang, A. Manchon, W.B. Mi, Y. Peng, X.X. Zhang. Phys. Rev. B 94, 2, 024428 (2016). https://doi.org/10.1103/physrevb.94.024428

[3] H. Fuse, N. Koshizaki, Y. Ishikawa, Z. Swiatkowska-Warkocka. Nanomaterials 9, 2, 198 (2019). https://doi.org/10.3390/nano9020198

[4] Y.N. Wu, D.B. Shieh, L.X. Yang, H.S. Sheu, R. Zheng, P. Thordarson, D.H. Chen, F. Braet. Materials 11, 12, 2572 (2018). https://doi.org/10.3390/ma11122572

[5] H. Okamoto, T.B. Massalski, L.J. Swartzendruber, P.A. Beck. Bull.Alloy Phase Diagrams 5, 6, 592 (1984). https://doi.org/10.1007/BF02868322

[6] J.A. Munoz, M.S. Lucas, L. Mauger, I. Halevy, J. Horwath, S.L. Semiatin, Y. Xiao, P. Chow, M.B. Stone, D.L. Abernathy, B. Fultz. Phys.Rev. B 87, 1, 014301 (2013). https://doi.org/10.1103/PhysRevB.87.014301

[7] J. Kangsabanik, R.K. Chouhan, D.D. Johnson, A. Alam. Phys. Rev. B 96, 10, 100201 (2017). https://doi.org/10.1103/PhysRevB.96.100201

[8] F. Calvo, N. Combe, J. Morillo, M. Benoit. J. Phys. Chem. C 121, 8, 4680 (2017). https://doi.org/10.1021/acs.jpcc.6b12551

[9] C. Srivastava, S. Chithra, K.D. Malviya, S.K. Sinha, K. Chattopadhyay. Acta Mater. 59, 16, 6501 (2011). https://doi.org/10.1016/j.actamat.2011.07.022

[10] A. Tymoczko, M. Kamp, O. Prymak, C. Rehbock, J. Jakobi, U. Schürmann, L. Kienle, S. Barcikowski. Nanoscale 10, 35, 16434 (2018). https://doi.org/10.1039/c8nr03962c

[11] М.Н. Магомедов. Кристаллография 62, 3, 487 (2017). [M.N. Magomedov. Crystallography Rep. 62, 3, 480 (2017).] https://doi.org/10.1134/S1063774517030142

[12] М.Н. Магомедов. Рос. нанотехнологии 14, 1-2, 19 (2019). [M.N. Magomedov, Nanotechnol.Rus. 14, 1-2, 21 (2019).] https://doi.org/10.1134/S1995078019010063

[13] М.Н. Магомедов. ФТТ 60, 5, 970 (2018). [M.N. Magomedov. Phys.Solid State 60, 5, 981 (2018).] https://doi.org/10.1134/S1063783418050190

[14] М.Н. Магомедов. ФТТ 45, 1, 33 (2003). [M.N. Magomedov. Phys. Solid State 45, 1, 32 (2003).]

https://doi.org/10.1134/1.1537405
[15] Л. Жирифалько. Статистическая физика твердого тела. Мир, М. (1975). 383 c. [L.A. Girifalco. Statistical Physics of Materials. J. Wiley and Sons Ltd., N.Y.(1973).]

[16] Е.Ф. Пичугин. Изв.вузов. Физика 6, 77 (1962). [E.F. Pichugin, Izvestiya Vysshikh Uchebnykh Zavedenii: Fizika 6. P. 77 (1962).] [in Russian]

[17] М.Н. Магомедов. Теплофизика высоких температур 44, 4, 518 (2006). [M.N. Magomedov. High Temperature 44, 4, 513 (2006).] https://doi.org/10.1007/s10740-006-0064-5

[18] М.Н. Магомедов. ФТТ 62, 7, 998 (2020). [M.N. Magomedov. Phys.Solid State 62, 7, 1126 (2020).] https://doi.org/10.1134/S1063783420070136

[19] М.Н. Магомедов. ФТТ 61, 11, 2169 (2019). [M.N. Magomedov. Phys. Solid State 61, 11, 2145 (2019).] https://doi.org/10.1134/S1063783419110210

[20] A. Karbasi, S.K.Saxena, R. Hrubiak. CALPHAD: Computer Coupling Phase Diagrams Thermochem. 35, 1, 72 (2011). https://doi.org/10.1016/j.calphad.2010.11.007

[21] Y. Nishihara, Y. Nakajima, A. Akashi, N. Tsujino, E. Takahashi, K.I. Funakoshi, Y. Higo. Am. Mineralogist 97, 8-9, 1417 (2012). https://doi.org/10.2138/am.2012.3958

[22] М.Н. Магомедов. ЖТФ 87, 4, 549 (2017). [M.N. Magomedov. Techn. Phys. 62, 4, 569 (2017).] https://doi.org/10.1134/S1063784217040156

[23] P.I. Dorogokupets, A.M. Dymshits, K.D. Litasov, T.S. Sokolova. Sci. Rep. 7, 41863, 1 (2017). https://doi.org/10.1038/srep41863

[24] М.Н. Магомедов. Поверхность. Рентген., синхротр., и нейтрон. исслед. 2, 103 (2018). [M.N. Magomedov. J. Surf. Investigation. X-ray, Synchrotron and Neutron Techniques 12, 1, 185 (2018).] https://doi.org/10.1134/S1027451018010299

[25] M.G. Pamato, I.G. Wood, D.P. Dobson, S.A. Hunt, L. Vočadlo. J. Appl.Crystallography 51, 2, 470 (2018). https://doi.org/10.1107/S1600576718002248

[26] V.K. Kumikov, Kh.B. Khokonov. J. Appl.Phys. 54, 3, 1346 (1983). https://doi.org/10.1063/1.332209

[27] Q. Jiang, H.M. Lu, M. Zhao. J. Phys.: Condens. Matter 16, 4, 521 (2004). https://doi.org/10.1088/0953-8984/16/4/001

[28] А.И. Фунтиков. Теплофизика высоких температур 41, 6, 954 (2003). [A.I. Funtikov. High temperature 41, 6, 850 (2003).] https://doi.org/10.1023/B:HITE.0000008344.89730.69

[29] H. Chamati, N.I. Papanicolaou, Y. Mishin, D.A. Papaconstantopouaolos. Surface Sci. 600, 9, 1793 (2006). https://doi.org/10.1016/j.susc.2006.02.010

[30] A.I. Karasevskii, V.V. Lubashenko. Low Temperature Phys. 35, 4, 275 (2009). https://doi.org/10.1063/1.3114592

[31] V.R. Selva, S.R. Kulkarni, S.K. Saxena, H.-P. Liermann, S.V. Sinogeikin. Appl. Phys.Lett. 89, 26, 261901 (2006). https://doi.org/10.1063/1.2422886

[32] R. Cherian, C. Gerard, P. Mahadevan, N.T. Cuong, R. Maezono. Phys. Rev. B 82, 23, 235321 (2010). https://doi.org/10.1103/PhysRevB.82.235321

[33] M. Popov, V. Churkin, D. Ovsyannikov, A. Khabibrakhmanov, A. Kirichenko, E. Skryleva, Y. Parkhomenko, M. Kuznetsov, S. Nosukhin, P. Sorokin, S. Terentiev, V. Blank. Diamond Rel. Mater. 96, 52 (2019). https://doi.org/10.1016/j.diamond.2019.04.033

[34] M. Mohr, A. Caron, P. Herbeck-Engel, R. Bennewitz, P. Gluche, K. Brühne, H.-J. Fecht. J. Appl. Phys. 116, 12, 124308 (2014). https://doi.org/10.1063/1.4896729 
[35] М.Н. Магомедов. Письма в ЖТФ 39, 9, 9 (2013). [M.N. Magomedov. Techn.Phys. Lett. 39, 5, 409 (2013).] https://doi.org/10.1134/S1063785013050076

[36] L. Liang, M. Li, F. Qin, Y. Wei. Phil. Mag. 93, 6, 574 (2013). https://doi.org/10.1080/14786435.2012.725950

[37] N.V. Galanis, I.N. Remediakis, G. Kopidakis. Mech. Mater. 67, 79 (2013).

https://doi.org/10.1016/j.mechmat.2013.07.019

[38] C.J. Bhatt, K. Kholiya. Indian J. Pure \& Appl.Phys. 52, 9, 604 (2014). http://nopr.niscair.res.in/handle/123456789/29354

[39] А.Е. Галашев, В.А. Полухин, И.А. Измоденов, О.А. Галашева. Поверхность. Рентген., синхротр., и нейтрон. исслед. 10, 60 (2007). [A.E. Galashev, V.A. Golukhin, I.A. Izmodenov, O.A. Galasheva. Poverkhnost' 10, 60 (2007).] [in Russian].

[40] B.J. Abdullah, M.S. Omar, Q. Jiang. Sādhanā 43, 11, 174 (2018). https://doi.org/10.1007/s12046-018-0956-1

[41] T.Y. Kim, J.E. Dolbow, E. Fried. Int. J. Solids Struct. 49, 26, 3942 (2012). https://doi.org/10.1016/j.jisolstr.2012.08.023

[42] М.Н. Магомедов. ФТТ 61, 1, 148 (2019). [M.N. Magomedov. Phys.Solid State 61, 1, 160 (2019).]

https://doi.org/10.1134/S1063783419010165

Редактор Т.Н. Василевская 